\title{
OPEN New solar energy-storage resource of plasmon-activated water solution with higher chemical potential
}

\author{
Chih-Ping Yang ${ }^{1,3}$, Shih-Hao Yu ${ }^{1,3}$, Fu-Der Mai ${ }^{1}$, Tai-Chih Kuo ${ }^{1 \bowtie}$ \& Yu-Chuan Liu ${ }^{1,2 \varpi}$
}

Nowadays, solar energy is the most environmentally friendly energy source to drive many chemical reactions and physical processes. However, the corresponding fabrication procedures for obtaining excellent energy-storage devices are relatively complicated and expensive. In this work, we report an innovative strategy on plasmon-activated water (PAW) serving as energy-storage medium from solar energy. The lifetime of the created energetic PAW solution from hot electron transfer (HET) on Au nanoparticles (AuNPs) illuminated with sunshine can last for 2 days, making the energy-storage system is practically available. Encouragingly, the energy-conversion efficiency from the solar energy in the PAW solution is ca. 6.7\%. Compared to conventional deionized (DI) water solution, the prepared metastable PAW solution exhibited distinctly higher chemical potential at room temperature. It demonstrates abilities in faster evaporation and enhancing chemical reactions, including hydrogen evolution reaction (HER) and oxygen evolution reaction (OER). Our proposed strategy on the simple and cheap energy-storage system based on prepared PAW utilizing solar energy is the first time shown in the literature.

Liquid water is the most abundant liquid on the earth and is recognized as the most environmentally friendly solvent in chemical reactions. Water's unique chemical and physical properties come from its flexible dynamic network of hydrogen bonds (HBs), in which HBs are broken and formed at equilibrium on a picosecond time scale $^{1,2}$. Because investigating water's local structure is still challenging ${ }^{3,4}$, well-known water properties are mainly based on free bulk water in spite of the original tetrahedral HB network being destroyed when water is confined in a nanosized environment or localized at interfaces ${ }^{5,6}$. These heterogeneous interactions result in corresponding changes in water's HB-dependent properties ${ }^{7,8}$. In fact, water deviated from the tetrahedral symmetry structure of bulk water would create disordered defect structure. As a result, it can reduce the size of water clusters. Compared to bulk bound water clusters, disordered water clusters with weak HBs have more free water molecules which can interact with other species to enhance the activity. As reported by Velasco-Velez et al. ${ }^{9}$, analysis of an ab initio molecular dynamic (AIMD) simulation revealed that $~ 50 \%$ of interfacial water molecules lie flat on a gold $(\mathrm{Au})$ surface with broken $\mathrm{HBs}$, and this population of broken $\mathrm{HB}$ molecules is substantially higher than the $22 \%$ found in bulk water.

On the other hand, due to increasing shortages of available energy in the world, the harvesting of solar energy has drawn enthusiastic interest due to it being a more environmentally friendly energy conversion process. For practical use, solar energy can be converted to heat, electricity, and energy-rich chemicals (solar fuel). Conversion to electricity and fuel is the most interesting and valuable because these forms of energy are more convenient to use $\mathrm{e}^{10-13}$. Correspondingly, energy-storage materials with enhanced energy-storage densities comprising highly stable devices are rapidly being developed. However, fabrication procedures for obtaining excellent energy-storage devices are relatively complicated. As shown in the literature, stable polymer phase-change materials embedded with $\mathrm{Fe}_{3} \mathrm{O}_{4}$-functionalized graphene nano-sheets were prepared serving as sunlight- and magnetic-driven energy conversion and storage nano-composites ${ }^{14}$. Also, to enable the synergistic coupling of electrochemical storage and light harvesting in a single electrode bias-free and solar-driven pseudocapacitors utilizing nanorod arrays of $\mathrm{ZnO} @ \mathrm{NiO}$ were proposed ${ }^{15}$.

${ }^{1}$ Department of Biochemistry and Molecular Cell Biology, School of Medicine, College of Medicine, Taipei Medical University, No. 250, Wuxing St., Taipei 11031, Taiwan. ${ }^{2}$ Cell Physiology and Molecular Image Research Center, Wan Fang Hospital, Taipei Medical University, Taipei, Taiwan. ${ }^{3}$ These authors contributed equally: Chih-Ping Yang and Shih-HaoYu. ${ }^{\varpi}$ email: tckuo@tmu.edu.tw; liuyc@tmu.edu.tw 
Because Au nanoparticles (NPs) possess well-defined localized surface plasmon resonance (LSPR) bands in the ultraviolet and near-infrared regions, they are commonly utilized in fields of surface-enhanced Raman scattering (SERS $)^{16}$, photothermal ablation of tumors ${ }^{17}$, and photochemical catalytic reactions ${ }^{18}$. Jia et al. ${ }^{19}$ reported the preparation of $\mathrm{Au} / \mathrm{CeO}_{2}$ microsphere photocatalysts through an aerosol spray and a study of their photocatalytic activities toward the aerobic oxidation of 1-phenylethanol under visible light based on hot electron transfer (HET). Meanwhile, Yoo et al. ${ }^{20}$ showed that electromagnetized AuNPs in the presence of specific electromagnetic field (EMF) conditions facilitate the efficient direct lineage reprogramming to induce dopamine neurons in vitro and in vivo. However, these chemical and biochemical reactions of AuNP-based efficient energy transfer occur transiently. The unutilized energy at the moment from excited or electromagnetized AuNPs cannot be preserved, meaning that the produced energies are wasted. Water's property and activity is critically dependent on the strength of hydrogen bonds (HBs) between water molecules. Innovative plasmon-activated water (PAW) was created utilizing HET on AuNPs with resonant illumination, as reported previously ${ }^{21}$. The created PAW with reduced HBs owns many distinct properties compared to bulk water. For examples, a smaller specific heat and a longer spin-lattice relaxation time. Recently, efficient and inexpensive catalysts are most developed for enhancing the efficiency on oxygen and hydrogen production. However, it is promising of an alternative approach to effective clean energy-relative reactions that employ PAW solutions, which are created from natural solar energy, with water molecules themselves possessing reduced HBs. The lifetime of HET is on the order of picoseconds, making the corresponding utilization limited and inconvenient. In this work, we suppose that the relatively large energetic barrier of HBs of bulk water could be overcome by utilizing solar-illuminated AuNPs to facilitate the dissociation of $\mathrm{H}_{2} \mathrm{O}$. Also, we demonstrate that the PAW solution with a higher chemical potential preserved from solar energy can serve as a new energy-storage resource to enhance chemical reactions and physical processes.

\section{Results and discussion}

Distinct properties of prepared PAW. From the viewpoint of thermodynamics, liquid water should be an excellent energy sink in utilizing solar energy, because its temperature is slowly raised when it is illuminated by sunshine and correspondingly being slowly decreased as it is cooled. However, most of the energy converted from solar energy is heat, and the dynamic equilibrium of $\mathrm{HBs}$ is on the level of picoseconds in water, making liquid water unavailable as an energy-storage resource when it is easily cooled to room temperature. On the other hand, HET can promote many chemical reactions, including the dissociation of hydrogen ${ }^{22}$ and activation of oxygen ${ }^{23}$. Interestingly, the dynamic processes of continuous and spontaneous forming, breaking, and rearranging of HBs occur at the picosecond scale, which is accordant with the lifetime of hot electrons ${ }^{24}$. Therefore, hot electrons may seize the moment to rapidly occupy breaking HBs and prevent them from reforming. This potential inspired us to utilize HET at excited AuNPs to create a metastable energetic PAW solution with intrinsically reduced HBs, which can serve as a new alternative energy-storage resource with a higher chemical potential from solar energy. Our previous $s t u d y^{21}$ reported that PAW can be created utilizing HET on AuNPs with resonant illumination of green LEDs. In this work, natural sunshine with full wavelengths, including plasmon resonance wavelength for the same AuNPs, is utilized to produce the similar HET on AuNPs and the subsequent PAW. Figure 1a shows the reaction glass cells for creating the PAW solutions $(0.1 \mathrm{M} \mathrm{KCl})$ exposed to sunlight. The light red color of the AuNP-coated ceramic rods (originally white color) was observed. This color is the characteristic one of AuNPs with photocatalytic activity, which is similar with our previous report (AuNP-coated ceramic particles), showing the creation of $\mathrm{PAW}^{21}$. As shown in the literature ${ }^{25,26}$, cations and anions impact the electronic structure of liquid water, resulting in distortions of the geometrical arrangements of water molecules. Therefore, two kinds of PAW solutions were prepared to examine the influence of electrolytes on HBs of the produced PAW solutions under solar irradiation in the presence of AuNPs. The first was a PAW solution in situ, in which $\mathrm{KCl}$ electrolytes were added to DI water before solar irradiation. The second was a PAW solution ex situ, in which $\mathrm{KCl}$ electrolytes were added to DI water after solar irradiation. Water's HBs are responsible for its fundamental abilities. Thus, this effect on the corresponding evaporation rate was first examined in ambient laboratory air. The left part of Fig. 1b demonstrates evaporation rates of different as-prepared aqueous solutions containing $0.1 \mathrm{M} \mathrm{KCl}$. Based on the colligative properties of ionic solutions, the vapor pressure of ionic solutions should be lower than that of a pure solvent. Thus, all experiments were performed on a platform of an orbital shaker for $30 \mathrm{~min}$ to accelerate the evaporation process. The measured evaporated masses in $30 \mathrm{~min}$ were $12.73 \pm 0.25$ (12.5, 13.0 and 12.7), $14.03 \pm 1.01(15.2,13.5$ and 13.4), and $13.77 \pm 1.27(15.2,11.4$ and 14.7) $\mathrm{mg}$ based on three replicated experiments (the following average data were also obtained using three replicated experimental results) for the DI water solution, PAW solutions in situ and ex situ, respectively. Magnitudes of the evaporation rates of the PAW solutions in situ and ex situ were respectively higher by ca. $10.2 \%$ and $8.2 \%$, compared to that of the DI water solution. The unique property of the high boiling point of water is ascribed to its strong HBs; thus, these increased evaporation rates observed in PAW solution systems with weak HBs are interesting. Moreover, the measured evaporation mass in 30 min was $12.97 \pm 0.76(13.5,13.3$ and 12.1) $\mathrm{mg}$ for the blank solution, which was obtained using similar experimental conditions (in the absence of AuNPs) as used to prepare the PAW solution in situ. This magnitude of the evaporation rate was slightly higher, by ca. $1.9 \%$, compared to that of the DI water solution, indicating that HET for weakening the HBs of water only occurred in the presence of AuNPs under solar irradiation. Moreover, for sunshine-irradiated experiments performed in open bottles, magnitudes of the evaporation rates of the PAW solutions in situ were higher by ca. $17.5 \%, 15.3 \%$ and $9.8 \%$ in the first, second and third hours, respectively, compared to those of the blank solutions, as shown in Fig. S1. These results confirm that the phenomena are photochemical processes. As shown in the literature, the potential of water is proportional to the natural logarithm of its vapor pressure according to the following equation ${ }^{27}$ :

$$
\mu=\mu_{\mathrm{o}}+\mathrm{RT} \ln \left(\mathrm{f} / \mathrm{f}_{\mathrm{o}}\right)
$$


a

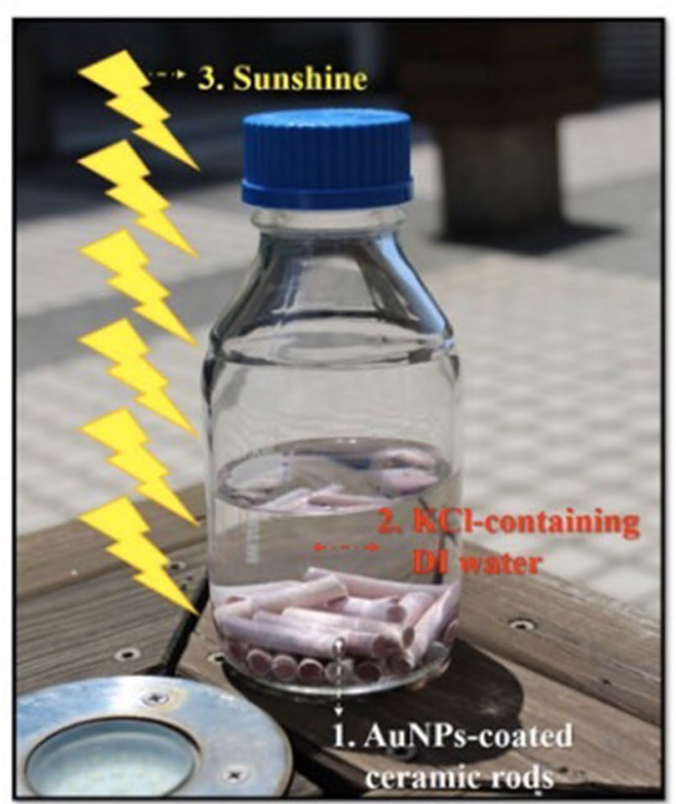

b

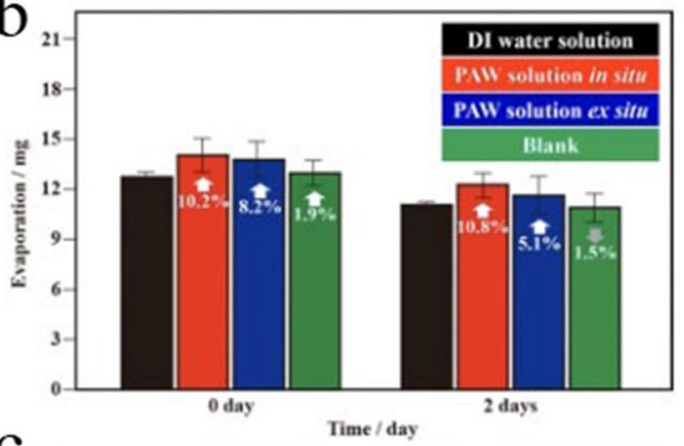

C

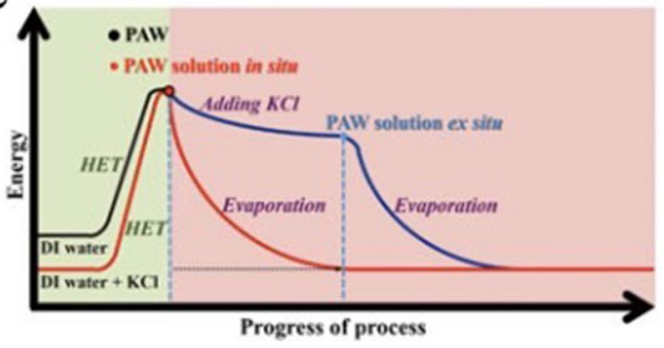

Figure 1. Reaction glass cell for creating plasmon-activated water (PAW) solutions under solar irradiation and corresponding evaporation rates of PAW-based solutions $(0.1 \mathrm{M} \mathrm{KCl})$ after exposure to sunlight for $3 \mathrm{~h}$. Deionized (DI) water-based solutions $(0.1 \mathrm{M} \mathrm{KCl})$ are demonstrated for reference. (a) Gold nanoparticle (AuNP)-coated ceramic rods in glass sample vials with $0.1 \mathrm{M} \mathrm{KCl-containing} \mathrm{DI} \mathrm{water} \mathrm{solutions} \mathrm{under} \mathrm{solar}$ irradiation: 1, AuNP-coated ceramic rods; 2, $0.1 \mathrm{M} \mathrm{KCl-containing} \mathrm{DI} \mathrm{water;} \mathrm{3,} \mathrm{sunshine.} \mathrm{(b)} \mathrm{Evaporation}$ rates in $30 \mathrm{~min}$ of the as-prepared and aged (2 days) PAW-based, DI water-based, and blank experiment-based solutions $(0.1 \mathrm{M} \mathrm{KCl})$; the blank solution was obtained by using experimental conditions similar to those for preparing the PAW solution in situ but using blank ceramic rods with no AuNP coating. (c) Schematic descriptions of the energy-progress of the process curve of PAW solutions in energy transfer for the dissolution and evaporation processes.

where $\mu$ and $\mu_{0}\left(\mathrm{~J} \mathrm{~mol}^{-1}\right)$ are defined as the potentials (i.e., thermodynamic activities or energies) at different vapor pressures of $\mathrm{f}$ and $\mathrm{f}_{0}$, respectively; $\mathrm{R}$ is the gas constant $\left(8.314 \mathrm{~J} \mathrm{~mol}^{-1} \mathrm{~K}^{-1}\right)$; and $\mathrm{T}$ is the temperature in $\mathrm{K}$. Compared to the DI water solution, the higher evaporation rate of the PAW solution means that the PAW solution possesses a higher vapor pressure than that of the DI water solution at room temperature. This suggests that the metastable PAW solution possesses higher activity similar to those of energy-rich chemicals with reduced HBs due to HET, which is in accordance with the increased activity observed for confined water ${ }^{28}$. The right part of Fig. $1 \mathrm{~b}$ demonstrates corresponding evaporation rates of different aqueous solutions containing $0.1 \mathrm{M} \mathrm{KCl}$ for 2 days after their preparation. In aging, the sealed glass sample bottles were placed in a dark atmosphere and in ambient laboratory air. The measured evaporation masses at $30 \mathrm{~min}$ were $11.07 \pm 0.12(11.2,11.0$ and 11.0), $12.27 \pm 0.74(11.7,12.0$ and 13.1), $11.63 \pm 1.72$ (10.9, 10.4 and 13.6), and $10.90 \pm 0.87(11.3,9.9$ and 11.5) $\mathrm{mg}$ for the DI water solution, the in situ and ex situ PAW solutions, and the blank solution, respectively. The magnitudes of the evaporation rates of the energetic PAW solutions in situ and ex situ were still higher by ca. $10.8 \%$ and $5.1 \%$, respectively, compared to that of the DI water solution after aging for 2 days. Similarly, the evaporation rate of the blank solution was close to that of the DI water solution. Conversion of the solar energy stored in the PAW solution means that is can be utilized for a couple of days after its creation; thus, it can possibly serve as a new energy-storage resource. Of course, the activity of the created PAW will decay with time.

The effects of adding electrolytes to the water on the corresponding properties of the prepared PAW solutions are another interesting finding. The increased evaporation rates compared to the DI water solution were more significant for the PAW solution in situ as observed in both fresh and aged samples. These phenomena were independent of the heat of the solution because using $\mathrm{KCl}$ with a positive heat of solution $\left(17 \mathrm{~kJ} \mathrm{~mol}^{-1}\right)$ and using $\mathrm{LiCl}$ with a negative heat of solution $\left(-38 \mathrm{~kJ} \mathrm{~mol}^{-1}\right)$ both demonstrated consistent results of higher evaporation rates in PAW solutions compared to them in DI water solutions, as shown in our previous study ${ }^{29}$. For the PAW solution ex situ, the reduced evaporation rate, compared to the PAW solution in situ, can be ascribed to the decreased vapor pressure due to the well-known colligative properties of ionic solutions, when $\mathrm{KCl}$ was added to the PAW. Some of the conserved energy from solar irradiation is utilized as the heat of solution for the dissolution of $\mathrm{KCl}$ in PAW. This release of chemical energy was accompanied with the formation of HBs in water molecules. Therefore, a new metastable PAW solution ex situ was formed with lower energy and stronger HBs compared to the PAW solution in situ. For the PAW solution in situ, the energy lost in dissolving $\mathrm{KCl}$ in DI water can easily be supplemented from HET under solar irradiation for $3 \mathrm{~h}$. Therefore, the PAW solution in situ is in a metastable state with a higher energy compared to the PAW solution ex situ. Pure PAW without electrolytes is in the same metastable state as the PAW solution ex situ under the same solar irradiation. The corresponding 
energy-progress process of the PAW solutions is illustrated in Fig. 1c. Throughout this work, unless stated otherwise, aqueous solutions were prepared with $0.1 \mathrm{M} \mathrm{KCl}$.

Moreover, similar photochemical experiments, as the one shown in Fig. 1a, were performed to demonstrate the phenomenon of HET based on electrolyte-free water. The measured zeta potentials of PAW were $-29.6 \pm 0.56$ and $-26.9 \pm 0.73 \mathrm{mV}$, respectively, 0 and 2 days after its creation; these values are close to electronically neutral DI water $(-1.63 \pm 0.41$ and $-1.58 \pm 0.29 \mathrm{mV}$, respectively, 0 and 2 days after its preparation). Meanwhile, compared to DI water, the intensities of hydroxyl free radicals measured by electron spin resonance spectroscopy ${ }^{21}$ decreased by $45 \pm 3.9 \%$ and $29 \pm 3.3 \%$, respectively, for as-prepared and 2 -day-aged PAW.

Calculating evaporation rates also disclosed a difference in $\mathrm{HBs}$ of the water solutions, which indicated different heat capacities. As expected, temperatures of both $0.1 \mathrm{M} \mathrm{KCl-containing} \mathrm{solutions} \mathrm{increased} \mathrm{with}$ the heating time (Fig. 2a). At temperatures of $>90^{\circ} \mathrm{C}$, the lines became flattened because the water was nearly boiling. The boiling point was $97.4 \pm 0.2^{\circ} \mathrm{C}$ for the DI water solution; while they were respectively reduced to $96.6 \pm 0.1$ and $96.4 \pm 0.2^{\circ} \mathrm{C}$ for the PAW solutions in situ and ex situ. Similarly, the boiling point of the blank solution $\left(97.8^{\circ} \mathrm{C}\right)$ was close to that of the DI water solution. It is recognized that HBs serve as a storehouse of energy. Instead of directly raising the temperature of the solution, part of heat is utilized for breaking HBs of water molecules. Due to fewer HBs remain at higher temperatures, thus, the heat capacity would decrease as the temperature increasing. In contrast, it would increase with rising temperatures. This phenomenon suggests that the added heat was utilized for breaking HBs of water molecules also for raising the temperature. Particularly, maintaining the degree of freedom between water molecules to prevent them from re-bonding. As a result that the PAW solutions can own intrinsically reduced HBs and could further prevent the re-bonding of HBs between water molecules. Therefore, it reduces the energy gap as the temperature rising. The specific heats of the PAW solutions in situ and ex situ were between 25 and $40^{\circ} \mathrm{C}$, which respectively demonstrate reduced values of 0.832 and 0.889 compared to the heat capacity of the DI water solution which was set to 1 (calculated from Fig. 2b). Because all of the experiments, using the same mass of water, were performed on the same heater with a constant heating rate the specific heats were inversely proportional to the slopes of lines, as shown in Fig. 2b. Compared to a general difference in specific heats of $<1 \%$ for DI water at low and high temperatures ${ }^{30}$, the $17 \%$ difference was indeed significant. This magnitude of the specific heat was slightly reduced by ca. $3.7 \%$ for the blank solution, compared to that of the DI water solution. These results are in agreement with the correlation of the water cluster size and heat capacity, in which the heat capacity of $\left(\mathrm{H}_{2} \mathrm{O}\right)_{21}$ is smaller than that of $\left(\mathrm{H}_{2} \mathrm{O}\right)_{50}$ at $27^{\circ} \mathrm{C}^{31}$. After aging for 2 days, the specific heats of the PAW solutions in situ and ex situ were between 25 and $40{ }^{\circ} \mathrm{C}$, which respectively demonstrated reduced values of 0.909 and 0.929 compared to the heat capacity of the DI water solution which was set to 1 (calculated from Fig. 2c). The magnitudes of the specific heats of the energetic PAW solutions in situ and ex situ were still reduced by ca. $9.1 \%$ and $7.1 \%$, respectively, compared to that in the DI water solution after aging for 2 days.

The evidence for weak HBs within pure PAW was discussed with deconvoluted Raman spectra in $\mathrm{O}-\mathrm{H}$ stretching vibrations in our previous repor $\mathrm{t}^{23}$. These $\mathrm{O}-\mathrm{H}$ stretching vibrations in Raman spectra are also sensitive to solutes in the water. Thus, the evidence for reduced HBs in PAW solutions was examined by diffusion averages of the nuclear magnetic resonance (NMR) relaxation time $\left(T_{1}\right)$ in this work (Fig. 3$)$. The strength of $\mathrm{HB}$ interactions among water molecules affected the spin-lattice relaxation time, $T_{1}$, which represents the time required for the longitudinal component of magnetization to recover its equilibrium value after application of a perturbing pulse sequence. Under magnetic field fluctuations for as-prepared solutions, $T_{1}$ values of the PAW solutions in situ and ex situ were $3.315 \pm 0.004$ and $3.281 \pm 0.041 \mathrm{~s}$, respectively, which were significantly longer than $3.230 \pm 0.055 \mathrm{~s}$ of the DI water solution. Similarly, with solutions aged for 2 days, $T_{1}$ values of the PAW solutions in situ and ex situ were $3.290 \pm 0.032$ and $3.240 \pm 0.004 \mathrm{~s}$, respectively, which were still longer than $3.139 \pm 0.022 \mathrm{~s}$ of the DI water solution. These analyses of NMR relaxation times suggested an intrinsic reduction of HB structures in the PAW solution. These energy-rich chemicals of reduced HBs show promise as an energy-storage resource for PAW solutions.

Distinctly electrochemical performance of prepared PAW. Water molecules exist in the form of water clusters in bulk water due to strong $\mathrm{HB}$ interactions. It was reported that the interaction energy of $\mathrm{H}_{3} \mathrm{O}^{+}-$ $\mathrm{OH}^{-}$is $46.9 \mathrm{~kJ} \mathrm{~mol}^{-1}$, and it increases approximately 2.5-times when $\mathrm{H}_{3} \mathrm{O}^{+}$associates with an additional four water molecules ${ }^{32}$. Based on these facts, creating PAW solutions with markedly weaker HB interactions could be advantageously used for efficient water splitting, compared to conventional DI water solutions. Potential applications of PAW solutions in OERs and HERs were evaluated by electrochemical linear sweep voltammetry (LSV). Figure 4a demonstrates the corresponding results of OERs in PAW-based and DI water-based solutions $(0.1 \mathrm{M} \mathrm{KCl})$. The onset potentials for the PAW solutions were markedly smaller (cathodic shifts) than that for the DI water solution, especially for the PAW solution in situ. This indicates that the required electrolytic energy for the OER was indeed reduced via weakening water's HBs. As the applied potential exceeded the onset potential, the current distinctly increased. At a vertex of $1.5 \mathrm{~V}$, the respective recorded currents were $1.060 \pm 0.165$ and $0.479 \pm 0.033 \mathrm{~mA}$ for the PAW solutions in situ and ex situ, which were ca. $220 \%$ and $40 \%$ higher than the $0.331 \pm 0.005 \mathrm{~mA}$ for the DI water solution. As shown in the literature, to increase the efficiency of OERs, the most common approaches have focused on new and cheap catalysts with different chemical compositions and structures $^{33,34}$. Investigations of the effect of the structure of reactant water itself on the corresponding efficiency of OERs have been less reported in the literature. Moreover, the increased efficiencies (depending on the recorded currents) of OERs performed in PAW solutions decreased with storage time (Fig. 4b). Similar currents for asprepared DI water solutions and those aged for 3 days mean that the HB structure in DI the water solution was stable during storage. However, the phenomenon of decreasing efficiencies for PAW solutions suggests that the broken HBs within water molecules recombine over time, resulting in a reduction in the efficiency of electrolytic 

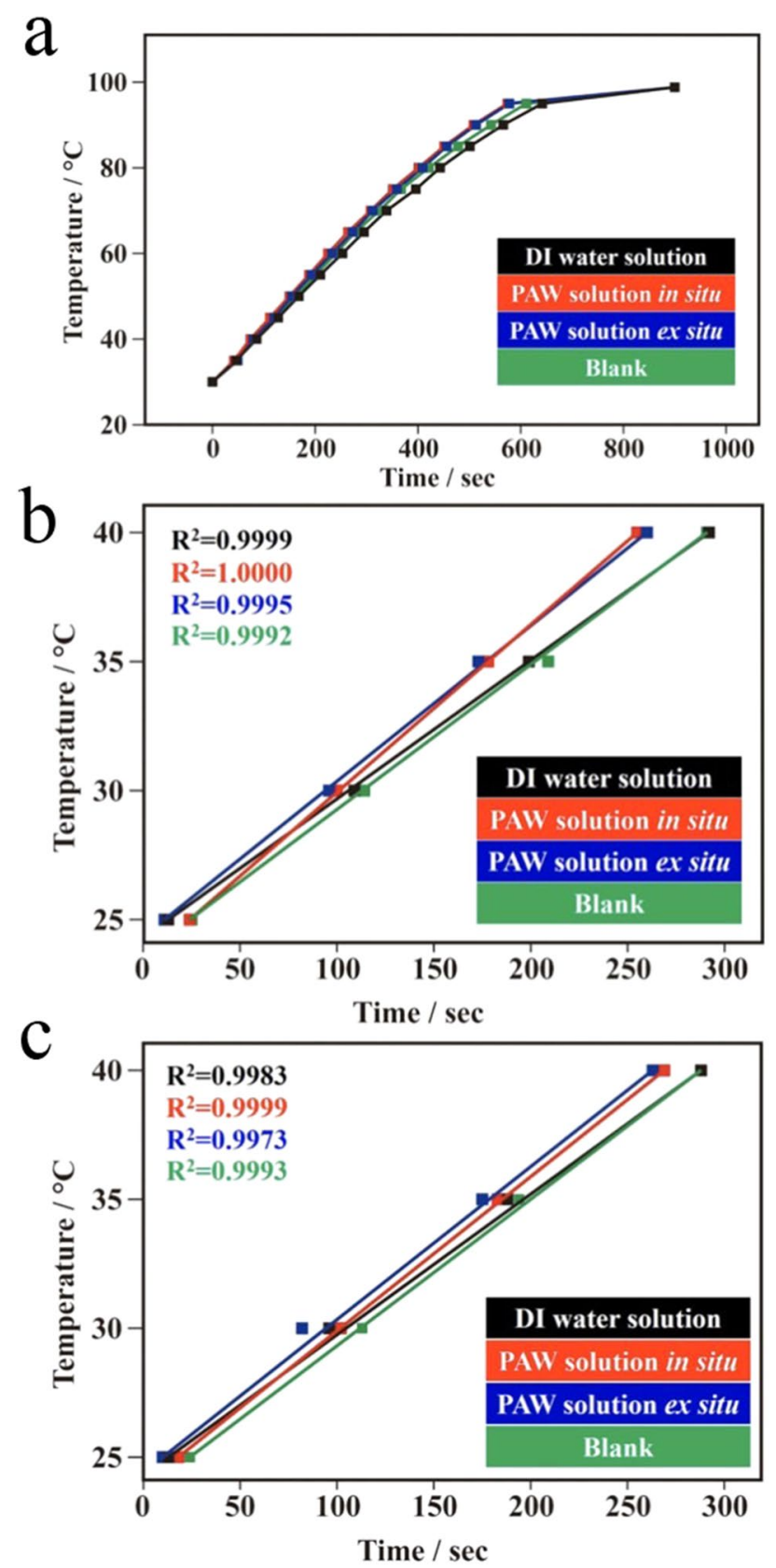

Figure 2. Specific heat (compared to the $0.1 \mathrm{M} \mathrm{KCl}$ deionized (DI) water solution) of plasmon-activated water (PAW)-based solutions $(0.1 \mathrm{M} \mathrm{KCl}$ ) after exposure to solar irradiation for $3 \mathrm{~h}$. (a) Rates of rising temperatures measured in the as-prepared PAW-based and DI water-based solutions $(0.1 \mathrm{M} \mathrm{KCl})$ with the same masses under a constant applied power. (b) The temperature-heating time dependence between room temperature and $40^{\circ} \mathrm{C}$ of the as-prepared PAW-based and DI water-based solutions. (c) The temperature-heating time dependence between room temperature and $40{ }^{\circ} \mathrm{C}$ of aged (for 2 days) PAW-based and DI water-based solutions. The blank solution was obtained using experimental conditions similar to those for preparing the PAW solution in situ but using blank ceramic rods without an AuNP coating.

water splitting. For the PAW solution ex situ, this increased efficiency in the OER was slight after aging for 1 day. Interestingly, for the PAW solutions in situ, these increased efficiencies in OERs of ca. $64 \%$ and $22 \%$ in magnitude were still significant after aging for 1 and 2 days, respectively. Again, as shown in Fig. S2, this increased efficiency of OERs for the as-prepared blank solution was slightly higher by ca. $9.2 \%$, compared to that for the DI water solution, indicating that HET for weakening HBs of water only occurred in the presence of AuNPs under solar irradiance. Figure 4c,d demonstrate the corresponding OERs performed in alkaline solutions containing $0.1 \mathrm{M} \mathrm{NaOH}$. Comparing Fig. 4c showing experiments performed in alkaline solutions with Fig. 4a showing experiments performed in neutral solutions, it was found that the recorded currents in alkaline solutions were significantly larger than those in neutral solutions. This is reasonable because an alkaline solution is favorable 

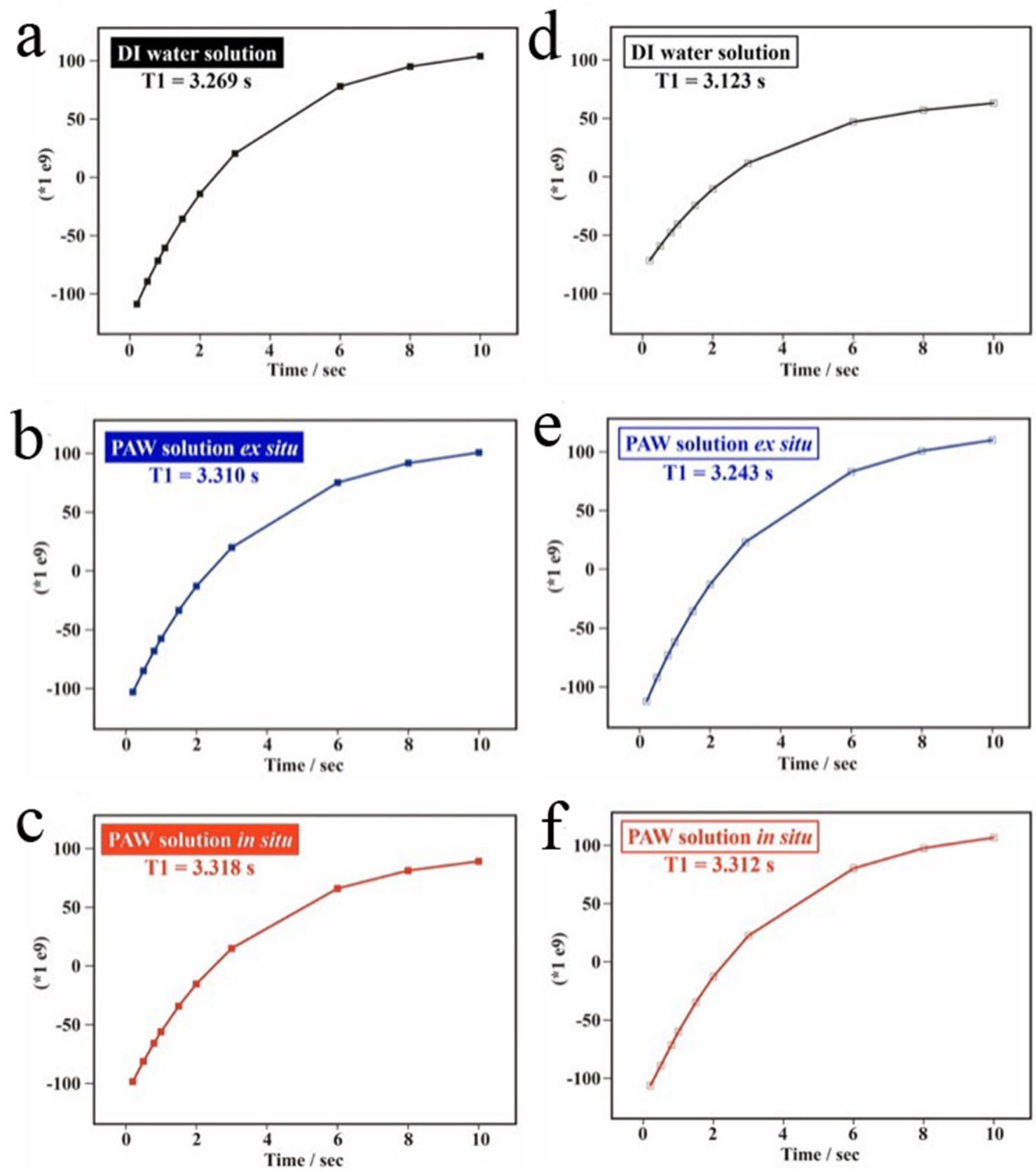

Figure 3. NMR- $T_{1}$ represents the time required for the longitudinal component of magnetization to recover to its equilibrium value after applying a perturbing pulse sequence. Spectra represent spectral signals as a function of the repetition time for (a) the deionized (DI) water solution $(0.1 \mathrm{M} \mathrm{KCl}$, as-prepared), (b) the plasmonactivated water (PAW) solution ex situ $(0.1 \mathrm{M} \mathrm{KCl}$, as-prepared), (c) the PAW solution in situ $(0.1 \mathrm{M} \mathrm{KCl}$, as-prepared), (d) the DI water solution ( $0.1 \mathrm{M} \mathrm{KCl}$, aged for 2 days), (e) the PAW solution ex situ $(0.1 \mathrm{M} \mathrm{KCl}$, aged for 2 days), and (f) the PAW solution in situ $(0.1 \mathrm{M} \mathrm{KCl}$, aged for 2 days).

for OERs. At a vertex of $1.5 \mathrm{~V}$, the recorded currents were $2.5400 \pm 0.1949$ and $2.2830 \pm 0.2621 \mathrm{~mA}$ for the PAW solutions in situ and ex situ, respectively, which were ca. $25 \%$ and $12 \%$ higher than the $2.0386 \pm 0.1708 \mathrm{~mA}$ of the DI water solution. Interestingly, for the PAW solutions in situ and ex situ, these respective increased efficiencies in OERs were of ca. $14 \%$ and $3.8 \%$ after aging for 3 days, as shown in Fig. 4 d. This increase was still significant for the PAW solution in situ.

Similarly, Fig. 5a shows the corresponding results of HERs in PAW-based and DI water-based solutions $(0.1 \mathrm{M}$ $\mathrm{KCl}$ ). Onset potentials of the PAW solutions exhibited significant anodic shifts compared to the DI water solution, especially for the PAW solution in situ. At a vertex of $-1.4 \mathrm{~V}$, the recorded currents were $-0.0793 \pm 0.0006$ and $-0.0494 \pm 0.0013 \mathrm{~mA}$ for the PAW solutions in situ and ex situ, respectively, which were ca. $120 \%$ and $35 \%$ higher than the $-0.0367 \pm 0.0015 \mathrm{~mA}$ of the DI water solution. As expected, the increased efficiencies (depending on the recorded currents) of HERs performed in the PAW solutions decreased with the storage time (Fig. 5b). 

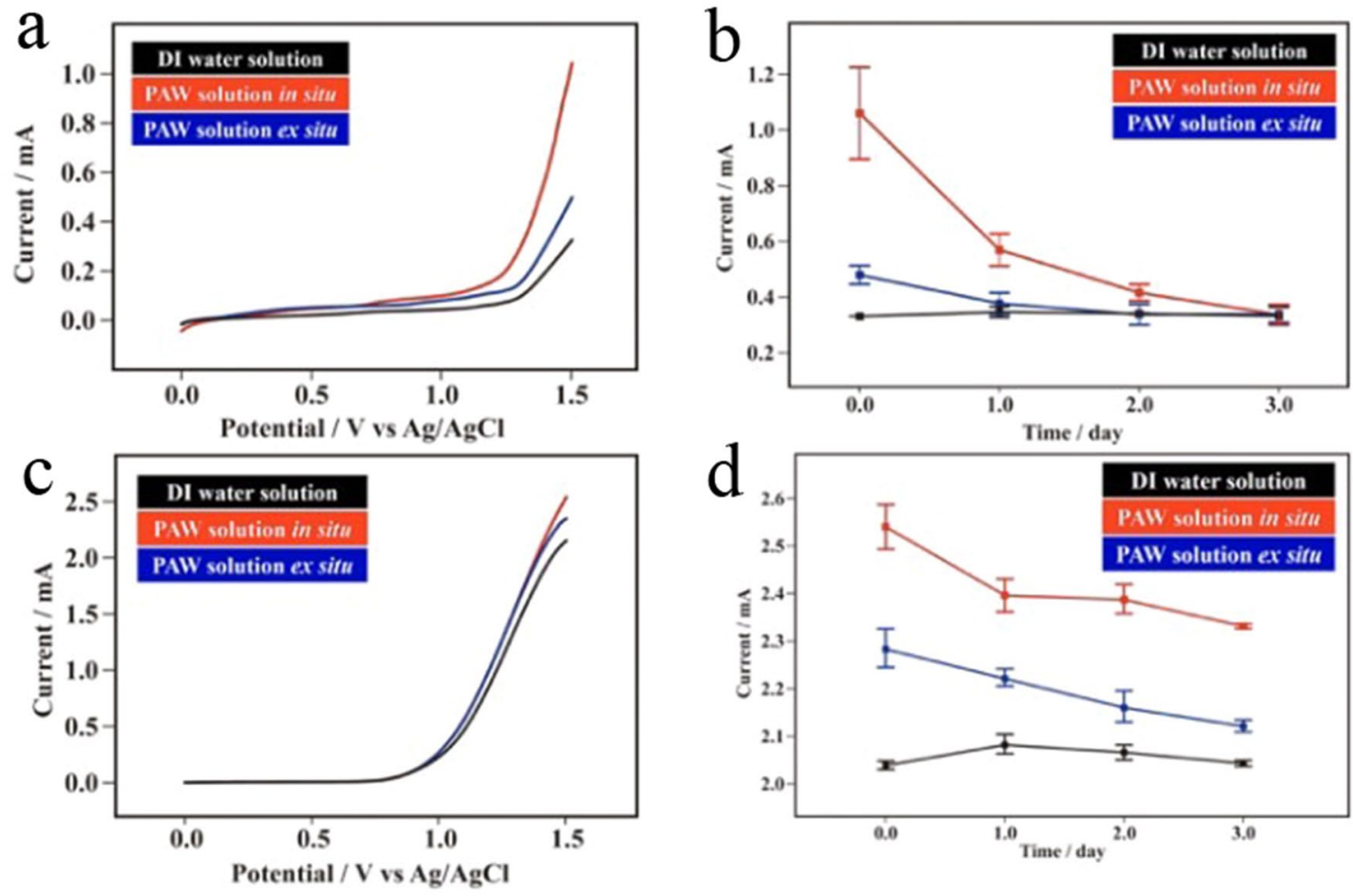

Figure 4. Linear sweep voltammetry (LSV) recorded on a planar Pt electrode for the oxygen evolution reaction (OER) in plasmon-activated water (PAW)-based and deionized (DI) water-based solutions. (a) LSV at scan rates of $0.05 \mathrm{~V} \mathrm{~s}^{-1}$ in the PAW solution in situ, the PAW solution ex situ, and the DI water solution (all containing $0.1 \mathrm{M} \mathrm{KCl}$ ). (b) OER currents at $1.5 \mathrm{~V}$ vs. $\mathrm{Ag} / \mathrm{AgCl}$ in the PAW solution in situ, the PAW solution ex situ, and the DI water solution (all containing $0.1 \mathrm{M} \mathrm{KCl}$ ) for $0,1,2$, and 3 days after their preparation. (c) LSV at scan rates of $0.05 \mathrm{~V} \mathrm{~s}^{-1}$ of the PAW solution in situ, the PAW solution ex situ, and the DI water solution (all containing $0.1 \mathrm{M} \mathrm{NaOH}$ ). (d) OER currents at $1.5 \mathrm{~V}$ vs. $\mathrm{Ag} / \mathrm{AgCl}$ in the PAW solution in situ, the PAW solution ex situ, and the DI water solution (all containing $0.1 \mathrm{M} \mathrm{NaOH}$ ) for $0,1,2$, and 3 days after their preparation.

Similar currents for the as-prepared DI water solution and that aged for 3 days mean that the HB structure in DI water solutions was stable during storage. For the PAW solution ex situ, this increased HER efficiency was slight after aging for 2 days. Interestingly, for the PAW solutions in situ, these increased efficiencies in HERs of ca. 60\%, $50 \%$ and $28 \%$ in magnitude were still significant after aging for 1, 2, and 3 days, respectively. Correspondingly, experimental results of evaporation rates, specific heats, OERs, and HERs suggest that the created metastable PAW solution in situ can serve as a new energy-storage material with energy-rich chemicals of reduced HBs.

Figure 6a demonstrates typical triangular voltammetric curves in the 5 th scan for anodic dissolution and cathodic redeposition of Au respectively onto Au substrates in an as-prepared PAW solution in situ, an as-prepared PAW solution ex situ, and an as-prepared DI water solution for reference. Basically, the anodic dissolution and cathodic redeposition of AuNPs on substrates were easier in PAW solutions (a reflection of the enhanced current), especially in the PAW solution in situ, than in the DI water solution. In the oxidation-reduction cycle (ORC) treatment for roughening the Au substrate, AuNPs were deposited on the Au substrate. Intrinsic activation energy is necessary for this electrochemical reaction. In our previous study ${ }^{21}$, it was proposed that the chemical potential of PAW is higher than that of DI water. Because the PAW solution is energetic, the actual required activation energy was correspondingly reduced, compared to the DI water solution. Therefore, enhanced currents were obtained in the PAW solutions. Compared to the DI water solution, the cathodic redeposition currents at ca. $0.28 \mathrm{~V}$ vs. $\mathrm{Ag} / \mathrm{AgCl}$ respectively increased by $13 \%$ and $9.7 \%$ for the PAW solutions in situ and ex situ. With up to 25 scans, as shown in Fig. 6b, these increases in cathodic redeposition currents at ca. $0.28 \mathrm{~V} \mathrm{vs.} \mathrm{Ag/AgCl} \mathrm{were}$ similar. Compared to the DI water solution, the cathodic redeposition currents respectively increased by $13 \%$ and 5.4\% for the PAW solutions in situ and ex situ. Similarly, as shown in Fig. 6c (the 5th scan) and 6d (the 25th scan) for solutions aged for 2 days, smaller increased currents were observed in experiments performed in the PAW solutions, especially in the PAW solution in situ, compared to the DI water solution. Other experimental results (Figs. 1, 2, 3, 4, 5 discussed before, and Figs. 7, 8 discussed later) all indicate that the property differences between the PAW solution and the DI water solution after aging for 2 days are significant. Therefore, this small difference in increment drops to around 2.2\%, as Fig. 6d, most likely can be ascribed to the solution difference. This also suggests that the PAW solution in situ is suitable for serving as an energy-storage resource. As described in the experimental section, the electrolytes of $\mathrm{KCl}$ were added in water before and after the creations of PAW for the PAW solution in situ and the PAW solution ex situ, respectively. Additional energy is necessary for the dissolution of $\mathrm{KCl}$ in water. Thus, the potential energy of the intrinsically energetic PAW is reduced for the preparation of the PAW solution ex situ. This results in the enhanced current being correspondingly reduced, as 

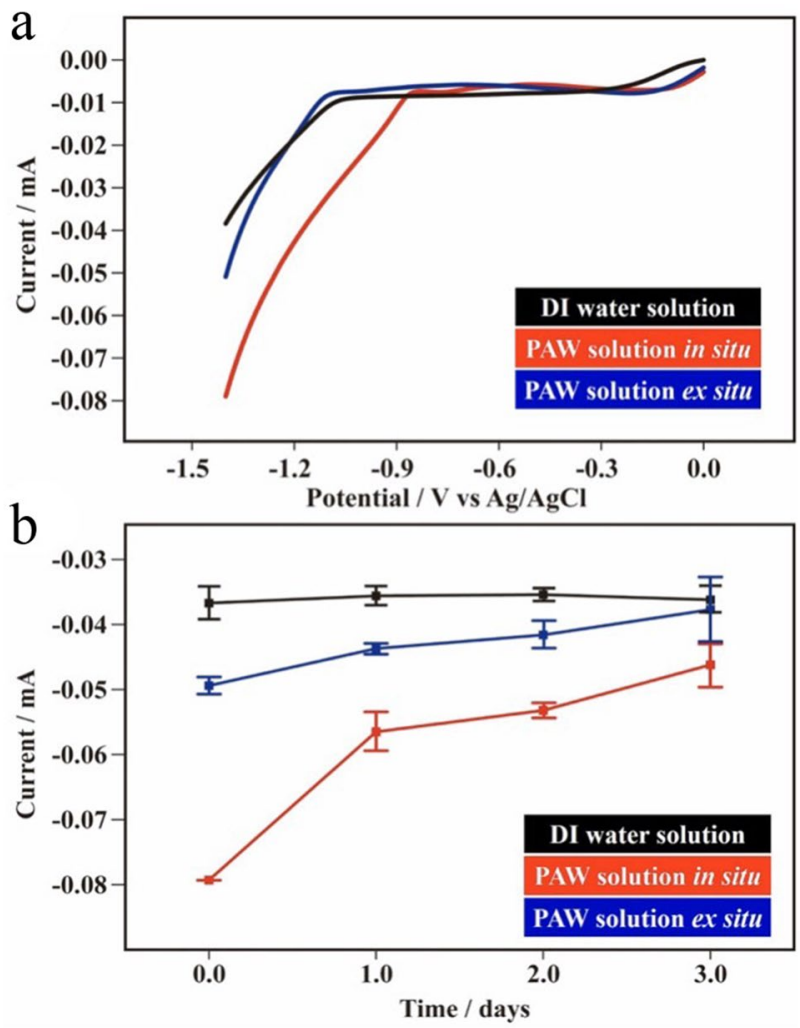

Figure 5. Linear sweep voltammetry (LSV) recorded on a planar Pt electrode for the hydrogen evolution reaction (HER) in plasmon-activated water (PAW)-based and deionized (DI) water-based solutions $(0.1 \mathrm{M}$ $\mathrm{KCl}$ ). (a) LSV at scan rates of $0.05 \mathrm{~V} \mathrm{~s}^{-1}$ in the PAW solution in situ, the PAW solution ex situ, and the DI water solution. (b) HER currents of at $-1.4 \mathrm{~V}$ vs. $\mathrm{Ag} / \mathrm{AgCl}$ in the PAW solution in situ, the PAW solution ex situ, and the DI water solution for $0,1,2$, and 3 days after their preparation.

compared to the PAW solution in situ. The higher currents at cathodic peaks observed in PAW solutions may be also ascribed to the higher diffusion coefficient and the higher electron transfer rate constant for electrochemical experiments performed in the PAW solution systems, as discussed below.

Figure 7a shows the $\mathrm{CVs}$ of $\mathrm{K}_{3} \mathrm{Fe}(\mathrm{CN})_{6}$ in the as-prepared PAW solutions and the as-prepared DI water solution. It can be observed that both the anodic and cathodic peak currents based on the PAW solutions were the highest ones, especially for the PAW solution in situ. Similarly, the potential energy of the intrinsically energetic PAW would be reduced in the dissolution of electrolytes for the preparation of the PAW solution ex situ. Thus, this results in the enhanced currents being correspondingly decreased, as compared to the PAW solution in situ. In accordance with the Randles-Sevcik equation ${ }^{35}$, the peak current is proportional to the square root of the diffusion coefficient. The calculated diffusion coefficients of $\mathrm{K}_{3} \mathrm{Fe}(\mathrm{CN})_{6}$ in the PAW solution in situ $\left(2.34 \pm 0.01 \times 10^{-6}\right.$ $\left.\mathrm{cm}^{2} \mathrm{~s}^{-1}\right)$ and in the PAW solution ex situ $\left(2.27 \pm 0.01 \times 10^{-6} \mathrm{~cm}^{2} \mathrm{~s}^{-1}\right)$ were ca. $17 \%$ and $14 \%$, respectively, higher than that in the DI water solution $\left(2.00 \pm 0.01 \times 10^{-6} \mathrm{~cm}^{2} \mathrm{~s}^{-1}\right)$. These results suggest that the PAW solution has a function of enhancing the diffusion ability of species in water. The reason might be attributed to the reduced size of hydrated $\mathrm{Fe}(\mathrm{CN})_{6}^{3-/ 4-}$ in the water. Within the DI water solution, hydration is associated with large water clusters due to the strong HB network of water. Contrarily, breaking the HB structure can reduce the size of water clusters in the PAW solution, resulting in higher mobility of $\mathrm{Fe}(\mathrm{CN})_{6}{ }^{3-/ 4-}-\mathrm{H}_{2} \mathrm{O}$. Figure $7 \mathrm{~b}$ shows the corresponding CVs of $\mathrm{K}_{3} \mathrm{Fe}(\mathrm{CN})_{6}$ in aged solutions. Also, the diffusion coefficients of $\mathrm{K}_{3} \mathrm{Fe}(\mathrm{CN})_{6}$ in the in situ PAW solution $\left(2.32 \pm 0.06 \times 10^{-6} \mathrm{~cm}^{2} \mathrm{~s}^{-1}\right)$ and in the PAW solution ex situ $\left(1.99 \pm 0.04 \times 10^{-6} \mathrm{~cm}^{2} \mathrm{~s}^{-1}\right)$ were ca. $25 \%$ and $7 \%$, respectively, still higher than that in the DI water solution $\left(1.86 \pm 0.03 \times 10^{-6} \mathrm{~cm}^{2} \mathrm{~s}^{-1}\right)$ after the samples had aged for 2 days. Moreover, as discussed in the supporting information (SI, Figs. S3-S5), electron transfer rate constants $\left(k_{s}\right)$ of $\mathrm{K}_{3} \mathrm{Fe}(\mathrm{CN})_{6}$ in the PAW solution in situ $\left(0.233 \pm 0.014 \mathrm{~s}^{-1}\right)$ and in the PAW solution ex situ $\left(0.226 \pm 0.015 \mathrm{~s}^{-1}\right)$ were ca. $6.9 \%$ and $3.7 \%$, respectively, higher than that in the DI water solution $\left(0.218 \pm 0.014 \mathrm{~s}^{-1}\right)$. Also, these constants for the PAW solution in situ $\left(0.257 \pm 0.008 \mathrm{~s}^{-1}\right)$ and for the PAW solution ex situ $\left(0.233 \pm 0.005 \mathrm{~s}^{-1}\right)$ were still ca. $18 \%$ and $7.4 \%$, respectively, higher than that for the DI water solution $\left(0.217 \pm 0.005 \mathrm{~s}^{-1}\right)$ after the samples had aged for 2 days. Moreover, as discussed in Fig. 7, including Figs. S3-S5, the higher diffusion coefficients and the higher electron transfer rate constants for experiments performed in PAW-based solutions contributed to the corresponding more-efficient OERs and HERs. In this work, the energy from the solar energy conversion is stored in the PAW solutions. This concept is quite different from the conventional situation. Two days later, the PAW solution can be retreated with the same process to again become an energetic PAW solution. That means that the PAW solution can be recycled. 

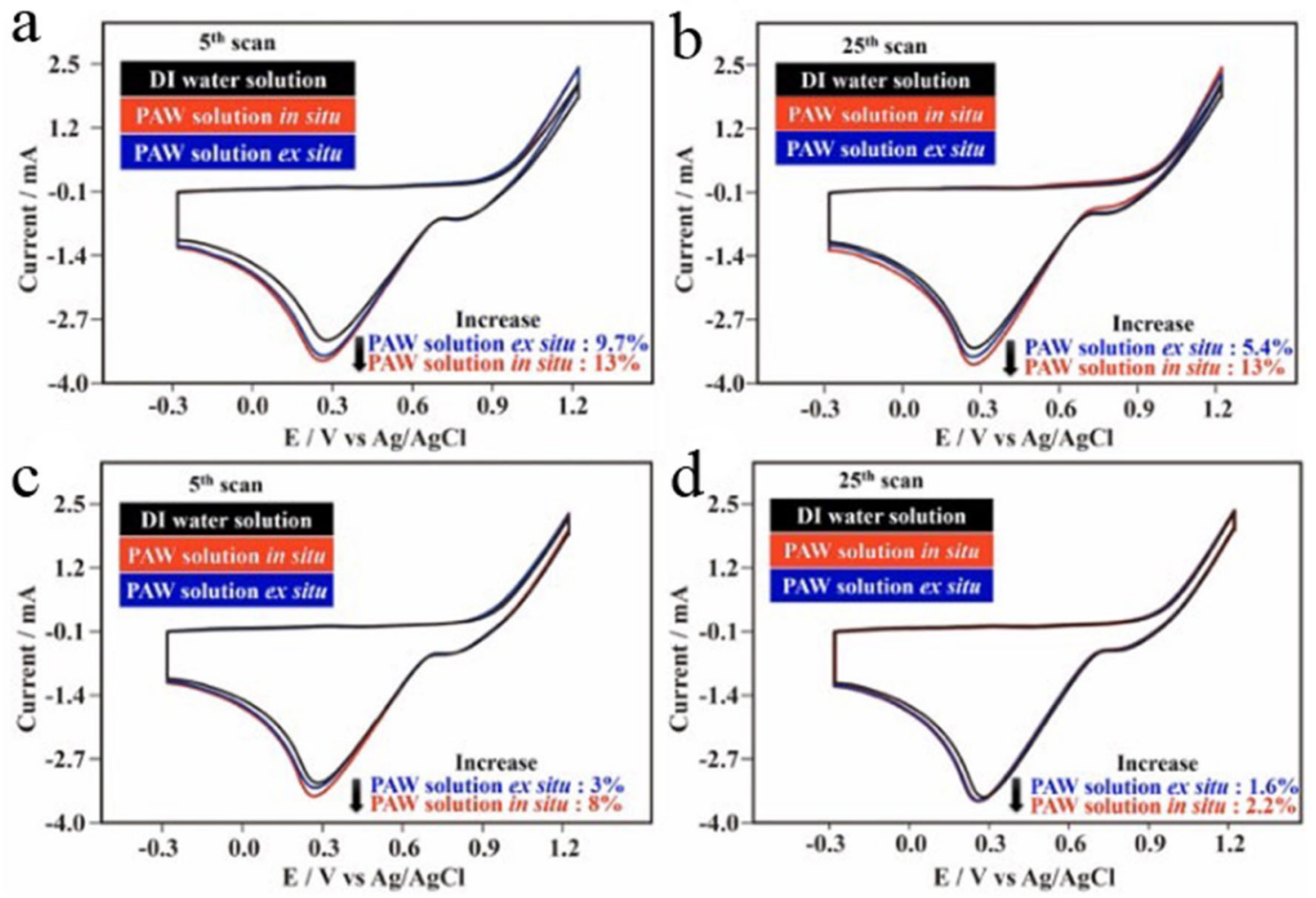

Figure 6. Cyclic voltammogram (CV) recordings on the same planar Au electrode showing the 5th (at the beginning) and the 25th (at the end) scans with oxidation-reduction cycle (ORC) treatments at $0.5 \mathrm{~V} \mathrm{~s}^{-1}$ to roughen the Au electrodes in plasmon-activated water (PAW)-based and deionized (DI) water-based solutions $(0.1 \mathrm{M} \mathrm{KCl})$. (a) The 5 th scans in the as-prepared solutions; (b) the 25th scans in the as-prepared solutions; (c) the 5 th scans in the solutions aged for 2 days; and (d) the 25th scans in solutions aged for 2 days.

The energy-conversion efficiency, $\eta$, in the preparation of a PAW solution under illumination of sunshine was estimated from the ratio of the energies required for breaking the HBs of bulk water and for raising the temperature of the PAW solution to that provided by solar energy, as defined below.

$$
\eta=\left(\mathrm{E}_{\mathrm{HB}} \mathrm{M}_{\text {water }}+\mathrm{ms} \Delta \mathrm{T}\right) /\left(\mathrm{P}_{\text {sunshine }} \mathrm{t}\right) \times 100 \%
$$

where the energy of hydrogen bonds, $\mathrm{E}_{\mathrm{HB}}$, of $20 \mathrm{~kJ} \mathrm{~mol}^{-1}$ was used. To obtain $250 \mathrm{~g}$ (or $250 \mathrm{~cm}^{3}$, when using a density of $1 \mathrm{~g} \mathrm{~cm}^{-3}$ ) of a PAW solution the moles of bulk water, $\mathrm{M}_{\text {water }}$, in which hydrogen bonds were broken, were calculated from the moles $(14 \mathrm{~mol})$ multiplied by the difference in values of DNHBW (degree of non-hydrogenbonded water) of DI water (21.29\%) and PAW water $(26.23 \%)^{21}$ under illumination by sunshine. The sunshine power $\left(\mathrm{P}_{\text {sunshine }}\right)$ of $1 \mathrm{~kW} \mathrm{~m}^{-2}$ was used ${ }^{36}$ and the 3 -h illumination time, $\mathrm{t}$, is $10,800 \mathrm{~s}$. The surface area of a $500 \mathrm{~mL}$ glass bottle is ca. $0.035 \mathrm{~m}^{2}$. The mass of the PAW solution is $250 \mathrm{~g}$. A specific heat, s, of $0.0042 \mathrm{~kJ} \mathrm{~g}^{-1}{ }^{\circ} \mathrm{C}^{-1}$ is used for the PAW solution. The measured temperature difference, $\Delta \mathrm{T}$, is $11^{\circ} \mathrm{C}$. Therefore, the energy-conversion efficiency of the preparation of the PAW solution under the illumination of sunshine was approximately $6.7 \%$ $((13.8+11.6) \mathrm{kJ} / 378 \mathrm{~kJ})$. Excluding the obtained sensible heat the energy-conversion efficiency from the solar energy conversion in the PAW solution is ca. $3.7 \%$. Moreover, in Eq. (2), $\mathrm{E}_{\mathrm{HB}} \mathrm{M}_{\text {water }}$ and $\mathrm{ms} \Delta \mathrm{T}$ are equivalent to the required energies on the latent and sensible heats, respectively. The required latent heat is roughly proportional to the increased evaporation rate of PAW compared to DI water. As discussed before in Fig. S1, for sunshineirradiated experiments performed in open bottles, magnitudes of the evaporation rates of the PAW solutions in situ were higher by ca. $17.5 \%, 15.3 \%$ and $9.8 \%$ in the first, second and third hours, respectively, compared to those of the blank solutions. Based on the calculated $\mathrm{E}_{\mathrm{HB}} \mathrm{M}_{\mathrm{water}}$ of $13.8 \mathrm{~kJ}$ for 3-h sunshine-irradiated experiment, the estimated $\mathrm{E}_{\mathrm{HB}} \mathrm{M}_{\text {water }}$ are ca. $5.67 \mathrm{~kJ}(13.8 \times(17.5 \% /(17.5+15.3+9.8) \%))$ and $10.6 \mathrm{~kJ}(13.8 \times((17.5+15.3) \% /$ $(17.5+15.3+9.8) \%)$ ) for 1 -h and 2 -h sunshine-irradiated experiments, respectively. Also, the measured temperature differences, $\Delta \mathrm{T}$, are 4.3 and $8.4^{\circ} \mathrm{C}$ for 1 - $\mathrm{h}$ and 2 - $\mathrm{h}$ sunshine-irradiated experiments, respectively. Thus, the energy efficiencies for the preparations of the PAW solutions under the illumination of sunshine are ca. 8.1\% $((5.67+4.52) \mathrm{kJ} / 126 \mathrm{~kJ})$ and $7.7 \%((10.6+8.82) \mathrm{kJ} / 252 \mathrm{~kJ})$ for the 1 -h and 2 -h sunshine-irradiated experiments, respectively. Excluding the obtained sensible heats the energy-conversion efficiencies from the solar energy conversions in the PAW solutions are ca. $4.5 \%$ and $4.2 \%$ for 1 -h and 2 -h sunshine-irradiated experiments, respectively. The energy-conversion efficiency uniquely developed in this work based on a water solution is comparable to other complicated systems, like the bifunctional NiFeSP/NF electrocatalyst implements unassisted solar-driven water splitting with a solar-to-hydrogen conversion efficiency of $\sim 9.2 \%{ }^{37}$. It is also comparable to that of the 


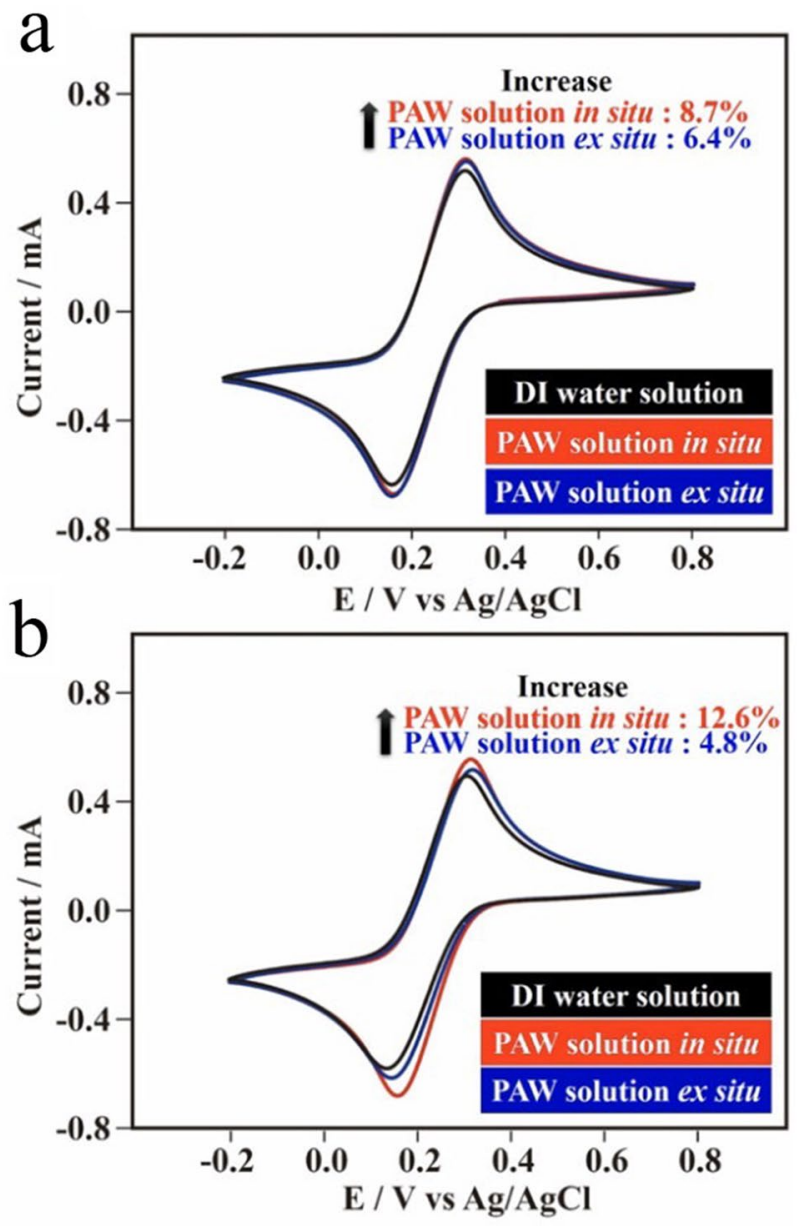

Figure 7. Cyclic voltammogram (CV) recordings on the same planar Au electrode showing the 3rd scans of the oxidation-reduction cycle (ORC) at $0.5 \mathrm{~V} \mathrm{~s}^{-1}$ in plasmon-activated water (PAW)-based and deionized (DI) water-based solutions $\left(50 \mathrm{mM} \mathrm{K}_{3} \mathrm{Fe}(\mathrm{CN})_{6}\right)$. (a) For the as-prepared solutions and (b) for solutions aged for 2 days.

$\mathrm{TiO}_{2} /$ dots/hibiscus/CdS photoanode with the poly(3,4-ethylenedioxypyrrole) @ $\mathrm{MnO}_{2}$ counter electrode in an aqueous polysulfide-silica gel electrolyte delivers a power conversion efficiency of $6.11 \%{ }^{38}$.

PAW can be created for water on AuNPs under resonant illumination ${ }^{21}$. In this work, an energetic PAW solution was obtained from HET of AuNPs excited by solar irradiation. Actually, this effect could also be observed on the rough surface of the Au substrate with AuNPs obtained from the ORC procedure under illumination from indoor fluorescent lamps. Figure $8 \mathrm{a}$ demonstrates the corresponding $\mathrm{CV}$ curves in the 5 th scans for anodic dissolution and cathodic redeposition of $\mathrm{Au}$, respectively, onto Au substrates in DI water with $0.1 \mathrm{M} \mathrm{KCl}$ under different degrees of illumination. Basically, the anodic dissolution and cathodic redeposition of AuNPs on substrates were easier in DI water solutions (a reflection of enhanced currents) with illumination, especially for more-powerful illumination with fluorescent lamps with no shadows on the substrate, than in a fully dark condition. Compared to the fully dark condition, cathodic redeposition currents at ca. $0.30 \mathrm{~V} v \mathrm{vs}$. Ag/ $\mathrm{AgCl}$ respectively increased by $10 \%$ and $6.9 \%$ for the environments with full illumination and with shadows on the substrate. With up to 25 scans, as shown in Fig. 8b, these increases in cathodic redeposition currents at ca. $0.30 \mathrm{~V} \mathrm{vs.} \mathrm{Ag} / \mathrm{AgCl}$ were similar but more significant because more AuNPs were deposited on the substrate with the increase in scanning. Compared to the fully dark condition, the cathodic redeposition currents respectively further increased by $14.1 \%$ and $7.6 \%$ for the environments with full illumination and with shadows on the substrate because more AuNPs were available for HET at higher scans.

The experimental results discussed above support that the relatively large energetic barrier of HBs of bulk water can be overcome by utilizing solar-illuminated AuNPs to facilitate the dissociation of $\mathrm{H}_{2} \mathrm{O}$. Moreover, the created PAW solution with a higher chemical potential preserved from solar energy can serve as a new energystorage resource to enhance water-related chemical reactions (like oxygen reduction reaction and production of $\mathrm{H}_{2} \mathrm{O}_{2}$ from water) and physical processes (like efficient water evaporation in desalination). This also resolves the concerning issue of the effective utilization of solar-driven HET with picosecond lifetimes.

In summary, we have successfully utilized the HET of solar energy-excited AuNPs to prepare PAW solutions with energy-rich chemicals as a new energy-storage resource for further chemical reaction. The PAW-based 

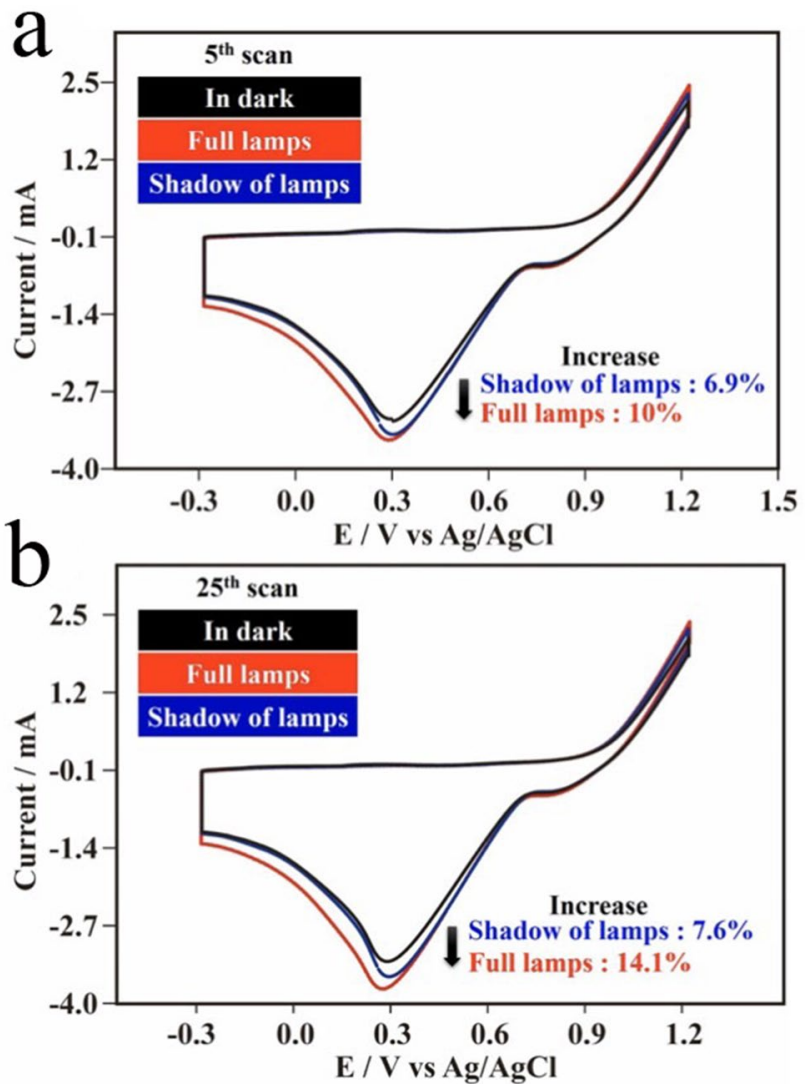

Figure 8. Cyclic voltammogram (CV) recordings of the same planar Au electrode showing the 5th (at the beginning) and the 25th (at the end) scans of the oxidation-reduction cycle (ORC) treatments at $0.5 \mathrm{~V} \mathrm{~s}^{-1}$ for roughening the Au electrode in deionized (DI) water-based solutions $(0.1 \mathrm{M} \mathrm{KCl})$ under different degrees of illumination from indoor fluorescent lamps. (a) The 5th scans in a completely dark condition, with full fluorescent lamps, and in the shadow of fluorescent lamps. (b) The 25th scans in a completely dark condition, with full fluorescent lamps, and in the shadow of fluorescent lamps.

energy-storage system is simple and practical. This developed strategy of effectively utilizing transient HET makes relative applications more convenient. The energy storage efficiency of the solar energy conversion of the PAW solution is ca. $6.7 \%$, which is comparable to other complicated systems shown in the literature. Compared to conventional DI water, the activity of the created energetic PAW solution can last for 2 days. The resulting metastable PAW solutions exhibited their ability to enhance HERs and OERs in fields of green energies. In particular, for the PAW solution in situ system, enhanced OER efficiencies of ca. 220\%, 64\%, and 22\% were measured for the as-prepared solution, and solutions aged for 1 and 2 days, respectively. These findings of a metastable PAW solution in situ with distinct activity for chemical reactions at room temperature, like energy-rich chemicals, are first presented in the literature. Further innovative applications of PAW in water-related fields and development of strategies for maintaining distinct activities of PAW are worthy of studies in the future.

\section{Methods}

Preparation of AuNP-coated ceramic rods. Twenty rinsed ceramic rods (with a diameter of $0.8 \mathrm{~cm}$ and a length of $4 \mathrm{~cm}$, also see Supporting Information (SI) for their components) were immersed in a sealed glass container with a 200-mL solution containing $50 \mathrm{ppm}$ AuNPs (ca. $10 \mathrm{~nm}$ ) in the dark for 1 day (see SI for the detailed preparation of AuNPs). After this process the AuNP concentration was reduced to ca. $42.7 \mathrm{ppm}$ because some AuNPs were adsorbed onto the ceramic rods. Therefore, the calculated quantity of AuNPs on each ceramic rod was ca. $7.3 \times 10^{-5} \mathrm{~g}\left(0.2 \mathrm{~L} \times(50-42.7) \mathrm{ppm}\left(\mathrm{mg} \mathrm{L}^{-1}\right) / 20\right)$. Then the AuNP-coated ceramic rods were rinsed thoroughly with deionized (DI) water, and finally dried in an oven at $120^{\circ} \mathrm{C}$ for 1 day. Before preparing the PAW solutions, the AuNP-coated ceramic rods were immersed and rinsed with DI water for several cycles until the $\mathrm{pH}$ values of the DI water were almost identical (ca. $\mathrm{pH} 7.15$ and water temperature at ca. $24^{\circ} \mathrm{C}$ ) before and after the rinsing process.

Preparation of the PAW solutions in situ and ex situ in sunshine. Eighteen AuNP-coated ceramic rods were placed in sample vials containing $250 \mathrm{~mL}$ of $0.1 \mathrm{M} \mathrm{KCl}$-containing DI water solutions. Then the sealed sample vials were placed in direct natural sunshine for $3 \mathrm{~h}$ around noon (from ca. 10:30 to 13:30) to create the PAW solutions in situ. After this solar irradiation, the $\mathrm{pH}$ of the solution slightly changed from 7.15 to 7.21 , and 
the temperature of the solution increased from 24 to $35^{\circ} \mathrm{C}$. The first examination of the created PAW solution in situ was immediately performed after the solution had been naturally cooled down to room temperature. Generally, this cooling process took less than $2 \mathrm{~h}$ after solar irradiation. Because all of the experiments based on the prepared PAW were performed at room temperature the created warm PAW under sunshine was further cooled down to room temperature. The PAW solution ex situ was created by following a similar process for creating the PAW solution in situ, but no electrolyte was added to the DI water before preparation of PAW in the sunshine. Instead, $\mathrm{KCl}$ was added to the created PAW immediately after removal from the sunshine to prepare the $0.1 \mathrm{M} \mathrm{KCl}$-containing PAW solution ex situ. Similarly, the first examination of the created PAW solution ex situ was immediately performed after the solution had been naturally cooled down to room temperature. To examine the purity of the prepared PAW solution, further inductively coupled plasma-mass spectrometer (ICPMS) analyses indicated that the concentrations of dissolved metals in the PAW solution after irradiation were ca. $0.48,23,14,17,7.6$, and $2.3 \mathrm{ppb}$ for $\mathrm{Au}, \mathrm{Na}, \mathrm{K}, \mathrm{Al}, \mathrm{Mg}$, and $\mathrm{Ca}$, respectively. These values were ca. $0.22,19,15$, 21,10 , and $3.6 \mathrm{ppb}$ for $\mathrm{Au}, \mathrm{Na}, \mathrm{K}, \mathrm{Al}, \mathrm{Mg}$, and $\mathrm{Ca}$, respectively, for a similar experiment performed in the dark as a reference. The increased concentration of $\mathrm{Au}$ is from the dissolved AuNPs on the AuNPs-coated ceramics due to hot electron transfer after direct sunlight ${ }^{21}$. The differences in concentrations of other metals are from the measuring fluctuations due to the low concentrations on ppb levels.

Received: 12 June 2020; Accepted: 2 November 2020

Published online: 30 November 2020

\section{References}

1. Laage, D., Stirnemann, G., Sterpone, F. \& Hynes, J. T. Water jump reorientation: from theoretical prediction to experimental observation. Acc. Chem. Res. 45, 53-62 (2012).

2. Laage, D. \& Hynes, J. T. A molecular jump mechanism of water reorientation. Science 311, 832-835 (2006).

3. Shultz, M. J., Vu, T. H., Meyer, B. \& Bisson, P. Water: a responsive small molecule. Acc. Chem. Res. 45, 15-22 (2012)

4. Paschek, D. \& Ludwig, R. Advancing into water's "no man's land“: two liquid states. Angew. Chem. Int. Ed. 53, 11699-11701 (2014).

5. David, J. G., Gierszal, K. P., Wang, P. \& Ben-Amotz, D. Water structural transformation at molecular hydrophobic interfaces. Nature 491, 582-585 (2012).

6. Chakraborty, S., Kumar, H., Dasgupta, C. \& Maiti, P. K. Confined water: structure, dynamics, and thermodynamics. Acc. Chem. Res. 50, 2139-2146 (2017).

7. Chakraborty, S., Kumar, H., Dasgupta, C. \& Maiti, P. K. Water at hydrophobic surfaces: weak hydrogen bonding and strong orientation effects. Science 292, 908-912 (2001).

8. Holt, J. K. et al. Fast mass transport through sub-2-nanometer carbon nanotubes. Science 312, 1034-1037 (2006).

9. Velasco-Velez, J. J. et al. The structure of interfacial water on gold electrodes studied by x-ray absorption spectroscopy. Science 346, 831-834 (2014).

10. Ponseca, C. S. Jr., Chabera, P., Uhlig, J., Persson, P. \& Sundstrom, V. Ultrafast electron dynamics in solar energy conversion. Chem. Rev. 117, 10940-11024 (2017).

11. Chen, J. et al. System development and environmental performance analysis of a solar-driven supercritical water gasification pilot plant for hydrogen production using life cycle assessment approach. Energ. Convers. Manage. 184, 60-73 (2019).

12. Jiaqiang, E. et al. Harmonic response analysis of a large dish solar thermal power generation system with wind-induced vibration. Sol. Energy 181, 116-129 (2019).

13. Zuo, H. et al. Catastrophic analysis on the stability of a large dish solar thermal power generation system with wind-induced vibration. Sol. Energy 183, 40-49 (2019).

14. Wang, W. et al. $\mathrm{Fe}_{3} \mathrm{O}_{4}$-functionalized graphene nanosheet embedded phase change material composites: efficient magnetic- and sunlight-driven energy conversion and storage. J. Mater. Chem. A 5, 958-968 (2017).

15. Zheng, X., Sun, Y., Qin, H. \& Ji, Z. Solar-charged pseudocapacitors: simultaneous conversion and storage of solar energy in ZnO@ $\mathrm{NiO}$ nanorod arrays. J. Alloys Compd. 781, 351-356 (2019).

16. Lim, D. K. et al. Highly uniform and reproducible surface-enhanced raman scattering from dna-tailorable nanoparticles with 1-nm interior gap. Nat. Nanotechnol. 6, 452-460 (2011).

17. Liu, Y. et al. Human induced pluripotent stem cells for tumor targeted delivery of gold nanorods and enhanced photothermal therapy. ACS Nano 10, 2375-2385 (2016).

18. Yan, L., Wang, F. \& Meng, S. Quantum mode selectivity of plasmon-induced water splitting on gold nanoparticles. ACS Nano 10, 5452-5458 (2016).

19. Jia, H., Zhu, X., Jiang, R. \& Wang, J. Aerosol-sprayed gold/ceria photocatalyst with superior plasmonic hot electron-enabled visible-light activity. ACS Appl. Mater. Interfaces 9, 2560-2571 (2017).

20. Yoo, J. et al. Electromagnetized gold nanoparticles mediate direct lineage reprogramming into induced dopamine neurons in vivo for Parkinson's disease therapy. Nat. Nanotechnol. 12, 1006-1014 (2017).

21. Chen, H. C. et al. Active and stable liquid water innovatively prepared using resonantly illuminated gold nanoparticles. ACS Nano 8, 2704-2713 (2014).

22. Zhou, L. C. et al. Aluminum nanocrystals as a plasmonic photocatalyst for hydrogen dissociation. Nano Lett. 16, 1478-1484 (2016).

23. Huang, Y. F. et al. Activation of oxygen on gold and silver nanoparticles assisted by surface plasmon resonances. Chem. Int. Ed. 53, 2353-2357 (2014).

24. Feldstein, M. J., Keating, C. D., Liau, Y. H., Natan, M. J. \& Scherer, N. F. Electronic relaxation dynamics in coupled metal nanoparticles. J. Am. Chem. Soc. 119, 6638-6647 (1997).

25. Yin, Z. et al. Cationic and anionic impact on the electronic structure of liquid water. J. Phys. Chem. Lett. 8, 3759-3764 (2017).

26. Gaiduk, A. P. \& Galli, G. Local and global effects of dissolved sodium chloride on the structure of water. J. Phys. Chem. Lett. 8, 1496-1502 (2017).

27. Van den Berg, C. \& Bruin, S. Water activity and its estimation in food systems: theoretical aspects. In Water Activity: Influences on Food Quality (eds Rockland, L. B. \& Stewart, G. F.) (Academic Press, New York, 1981).

28. Zhou, H. X. Helix formation inside a nanotube: possible influence of backbone-water hydrogen bonding by the confining surface through modulation of water activity. J. Chem. Phys. 127, 245101-245104 (2007).

29. Yang, C. P. et al. Effective energy transfer via plasmon-activated high-energy water promotes its fundamental activities of solubility, ionic conductivity, and extraction at room temperature. Sci. Rep. 5, 18152 (2015).

30. Miller, J. R., Schorr, G. R. Jr. \& Yaws, C. L. Correlation constants for liquids-heat capacities. Chem. Eng. 83, 129-131 (1976). 
31. Douady, J., Calvo, F. \& Spiegelman, F. Effect of an ionic impurity on the caloric curves of water clusters. Eur. Phys. J. D 52, 47-50 (2009).

32. Lee, H. M. \& Kim, K. S. Dynamics and structural changes of small water clusters on ionization. J. Comput. Chem. 34, 1589-1597 (2013).

33. Song, F. \& Hu, X. Exfoliation of layered double hydroxides for enhanced oxygen evolution catalysis. Nat. Commun. 5, 4477 (2014).

34. Park, J. et al. iridium-based multimetallic nanoframe@nanoframe structure: an efficient and robust electrocatalyst toward oxygen evolution reaction. ACS Nano 11, 5500-5509 (2017).

35. Gosser, D. K. Jr. Cyclic Voltammetry Simulation and Analysis of Reaction Mechanisms (VCH, Weinheim, 1993).

36. El-Sharkawi, M. A. Electric Energy 87-88 (CRC Press, Cambridge, 2005).

37. Xin, Y., Kan, X., Gan, L. Y. \& Zhang, Z. Heterogeneous bimetallic phosphide/sulfide nanocomposite for efficient solar-energydriven overall water splitting. ACS Nano 11, 10303-10312 (2017).

38. Das, A., Deshagani, S., Kumar, R. \& Deepa, M. Bifunctional photo-supercapacitor with a new architecture converts and stores solar energy as charge. ACS Appl. Mater. Interfaces 10, 35932-35945 (2018).

\section{Acknowledgements}

The authors thank the Ministry of Science and Technology (MOST) of ROC and Taipei Medical University for their financial support.

\section{Author contributions}

Y.C.L. and T.C.K. conceived the idea of the project and wrote the manuscript. C.P.Y., S.H.Y., T.C.K. and Y.C.L. designed the experiments. C.P.Y., S.H.Y. and F.D.M. performed the experiments. C.P.Y., S.H.Y. and F.D.M. analyzed the experimental data. C.P.Y., S.H.Y., T.C.K. and Y.C.L. discussed the results and commented on the paper.

\section{Competing interests}

The authors declare no competing interests.

\section{Additional information}

Supplementary information is available for this paper at https://doi.org/10.1038/s41598-020-77815-3.

Correspondence and requests for materials should be addressed to T.-C.K. or Y.-C.L.

Reprints and permissions information is available at www.nature.com/reprints.

Publisher's note Springer Nature remains neutral with regard to jurisdictional claims in published maps and institutional affiliations.

(c) (1) Open Access This article is licensed under a Creative Commons Attribution 4.0 International License, which permits use, sharing, adaptation, distribution and reproduction in any medium or format, as long as you give appropriate credit to the original author(s) and the source, provide a link to the Creative Commons licence, and indicate if changes were made. The images or other third party material in this article are included in the article's Creative Commons licence, unless indicated otherwise in a credit line to the material. If material is not included in the article's Creative Commons licence and your intended use is not permitted by statutory regulation or exceeds the permitted use, you will need to obtain permission directly from the copyright holder. To view a copy of this licence, visit http://creativecommons.org/licenses/by/4.0/.

(c) The Author(s) 2020 